\title{
Algunos rasgos y particularidades de la polarización territorial de la producción de contenidos Web en México
}

\author{
DJAMEL TOUDERT*
}

\begin{abstract}
A territorial polarisation of the World Wide Web production has emerged from the contexts that transform the Internet in a leitmotif of the socioterritorial appropriation of different information and communication technologies. The territorial asymmetry between production and consumption of Web contents is transformed into a theoretical challenge that cannot take into account the conceptual development of Geography and their territorial disciplines. In this paper, the empiric exploration of the Web production in Mexico leads us to unravel a Web that is enrolled in the traditional polarisation of territorial development. The findings of this paper puts forward some ideas that clarify the connection between the territorial agglomeration of the Web players and the decentralisation of its contents.
\end{abstract}

Keywords: World Wide Web, contents, internet, ICT, Mexico, federal entities, socio-teritorial development

\section{Resumen}

La polarización territorial de la producción de contenidos World Wide Web surge en un contexto en el que Internet se advierte como leit motiv de la apropiación socioterritorial de las tecnologías de la información y comunicación. Esta polarización se convierte en un reto teórico que no logra ser advertido por el desarrollo conceptual de la Geografía y las disciplinas territoriales. En este artículo, a partir de una exploración empírica de la producción de contenidos Web en México, se advierte un posible posicionamiento conceptual de una Web enlistada en la tradicional polarización del desarrollo territorial. Los resultados de esta investigación aclaran la conexión entre aglomeración territorial de los actores de la Web y la centralización de sus contenidos.

Palabras Claves: World Wide Web, contenidos, internet, TIC, México, Entidades federativas, Desarrollo socioterritorial.

"Instituto de Investigaciones Sociales, Universidad Autónoma de Baja California. Correo-e: toudert@uabc.mx y ceagi2004@yahoo.com 


\section{Introducción}

Desde su puesta en marcha por Tim Berners-Lee en 1991, la World Wide Web (www) tuvo un crecimiento espectacular, volviéndose rápidamente, después del correo electrónico, en la red de acceso público con más usuarios en el conjunto de las redes de la Internet. ${ }^{1}$ En una escala global, la www siempre fue caracterizada por una creciente difusión con el incremento del volumen de la información ofertada y el número de usuarios que interactúan de una forma u otra con sus contenidos. Sin embargo, desde sus orígenes, la Web estuvo marcada por flagrantes asimetrías entre países desarrollados y emergentes en la creación de contenidos y en el nivel de accesibilidad de las poblaciones. En este sentido, la polarización de la Web a escala de países es un hecho bien difundido -entre otros- con la publicación de estadísticas de dominios Web y usuarios por parte de los órganos de gobierno de la Internet y algunas consultorías dedicadas a labores de inteligencia en el mercado de la "nueva economía".

Pasando del nivel de países al de entornos territoriales intranacionales, nos encontramos con pocos trabajos enfocados a los mismos acontecimientos; en lo general, la literatura en cuestión se centra en la exploración del caso estadounidense. O sea, con relación el tema de la polarización socioterritorial de la producción Web, es indispensable expandir el campo de la investigación a otros países con la finalidad de sustentar un marco conceptual en la diversidad de los contextos.

La presente contribución se inscribe en una dinámica de exploración y de análisis en el nivel de las entidades federativas con el interés fundamental de buscar, desde la perspectiva de la geografía, una posible inserción conceptual de los fenómenos de aglomeración que prevalecen en la producción de la Web en México. Lo anterior toma posición en un supuesto de incompatibilidad del discurso de la sociedad de la información con la

\footnotetext{
${ }^{1}$ Para referir a la World Wide Web usaremos en este artículo las abreviaciones www y Web. Conforme a las explicaciones detalladas en la sección "Procedimientos y mecánica de generación de datos", el concepto de la Web mexicana se aplica a la totalidad de los dominios Web registrados bajo la bandera .MX y cuyos propietarios son ubicados en México. En el mismo orden de ideas, "la produccion mexicana de la Web" alude exclusivamente a esos dominios producidos. De una manera general, los términos "polarización" y "aglomeración" se aplican también a los mismos dominios producidos y registrados en México.

${ }^{2}$ Entre ellas puede citarse el Internet Systems Consortuim, Internet Traffic Report, Netcraft y Nua Internet Surveys.
} 
lógica del desarrollo sustentada en la polarización territorial de la producción de contenidos Web.

El trabajo consta de cuatro secciones: en la primera tratamos de encontrar un posicionamiento para la polarización de la Web mexicana en el marco de los grandes ejes del discurso disciplinario; en la segunda se presentan los métodos e instrumentos que brindaron un sustento empírico para las afirmaciones y supuestos desarrollados; en la tercera se lleva a cabo una caracterización de la polarización por sus rasgos socioterritoriales más importantes, y finalmente, en la última sección se presenta la discusión de los hallazgos en un marco comparativo, destacando: la singularidad de la polarización mexicana, la necesidad conceptual de ampliar la exploración a otros países emergentes con énfasis en la posible implicación de las externalidades de tipo informativo, y las estrategias de manejo organizacional en la centralización de contenidos.

\section{La búsqueda de un posicionamiento conceptual para la polarización de la www mexicana}

La polarización de la producción de la www constituye un interés interdisciplinario que destaca una creciente convergencia temática entre las ciencias computacionales y las ciencias humanas y sociales.

A pesar de la posición estratégica que toma el componente territorial en la construcción de los discursos de la apropiación de las Tecnologías de la Información y las Comunicaciones (TIC), la contribución de la geografía y sus disciplinas sigue siendo marginal y de poco impacto en el campo de las ciencias sociales en los países emergentes como el nuestro (Toudert y Buzai, 2004). De manera general, se registra una cierta coincidencia entre la polarización de la Web y la centralización de su investigación académica principalmente en Estados Unidos.

\subsection{Los ejes centrales de construcción del discurso disciplinario}

Dentro del debate académico en el seno de la geografía y sus disciplinas territoriales, son las contribuciones de los empiristas críticos las que más enfocaron a la Web como una plataforma de observación y de análisis para el desarrollo de un posicionamiento con relación a la apropiación socioterritorial de las TIC (Moss y Townsend, 1997; Kolko, 2000; Zook, 2005). Los estudios realiza- 
dos por los empiristas de primera generación, especialmente en las divisiones territoriales internas de Estados Unidos, lograron resaltar un abordaje respaldado en la metropolización de la infraestructura, usos y habilidades en la adopción y difusión de las TIC en general y la Internet en particular (Moss y Townsend, 2000; Dodge y Kitchin, 2001; Forman et al., 2003; Greenstein, 2006).

Conforme a la creciente legitimación de la investigación de la Web, empieza apenas a perfilarse en la actualidad una segunda generación de empiristas no menos crítica, pero con una aparente disponibilidad para examinar los acontecimientos desde un enfoque multidimensional (Forman et al., 2005; Greenstein, 2006; Blum y Goldfarb, 2005).

Globalmente, asistimos a la consolidación de un doble abordaje teórico que parece estructurar la parte más sustantiva del discurso disciplinario de la apropiación de las TIC. En el primero prevalece un posicionamiento desarrollado, generalmente, fuera de las esferas académicas, derivado de la visión de una geografía sin fricción sustentada en el determinismo tecnológico, la trascendencia de las TIC y la sustitución del espacio real por el espacio virtual (Gaspar y Glaeser, 1998; Cairncross, 1997 y 2001). En una posición diametralmente opuesta se encuentren las tesis del desarrollo de las TIC que se respaldan, generalmente, en la teoría de la sociedad de redes inspirada -entre otros- por los trabajos de Castells. Los promotores de esas tesis coinciden en que el proceso de apropiación de las TIC obedece a una distribución jerárquica de la infraestructura, usos y habilidades encaminada a consolidar la dominación metropolitana y urbana (Moss y Townsend, 1997; Kolko, 2000; Graham y Marvin, 2001; Zook, 2005). En este último entorno conceptual, marcado -entre otrospor la aportación de empiristas críticos de primera generación y particularmente los que tuvieron un acercamiento con la perspectiva de la "brecha digital", se sostiene que la evolución a la baja de los costos de adopción en cuencas de consumo pone en aparente ventaja a las localidades caracterizadas por fuertes densidades demográficas y amplias dinámicas económicas (Forman et al., 2005; Greenstein, 2006). Sin embargo, en el caso de que el posicionamiento anterior logre caracterizar los rasgos generales de la apropiación socioterritorial de las TIC, no deja de presentar una aparente debilidad para fundamentar las lógicas de aglomeración de los actores del desarrollo.

Para dar al posicionamiento anterior un alcance explicativo en el marco de las dinámicas de aglomeración, recientemente los empiristas críticos de segunda generación plantearon la necesi- 
dad de un enfoque epistemológico centrado en la interacción entre la dominación metropolitana y las coincidencias encontradas en la diversificación y especialización de la actividad económica (Forman et al., 2005). El modelo explicativo propuesto encuentra sentido en el marco de la segmentación de las TIC en herramientas con propósitos generales de una integración participativa (o pasiva) de cara a una adopción más especializada para el manejo estructural y organizacional (Bresnahan y Trajtenberg, 1995).

Desde otra perspectiva puede decirse que estas lógicas de aglomeración son fundamentadas también en los tipos de externalidades de red que pueden resultar igual de importantes para el entendimiento de las lógicas de atracción local y regional (Saxenian, 2000). En este sentido, además de los enfoques de la competitividad imperfecta y la interacción estratégica explorados por la geografía económica, Cahuc, Kempf y Verdier (2001) introducen el papel de las interacciones sociales, expresadas en términos de externalidades de tipo informativo que caracterizan el comportamiento de adopción y difusión de las TIC. Según esta perspectiva, tendremos dos lógicas de aglomeración: en la primera, las externalidades son producto de la retroalimentación positiva y el aplazamiento de las preferencias individuales que concurren para mejorar progresivamente la atracción local y regional (Capello y Nijkamp, 1995; Zimmermann, 2001); mientras que la segunda se sustenta en un mimetismo originado en la incertidumbre con relación a los posibles resultados de localización, o sea, ubicaciones en cascada respaldadas en la toma de decisión de los demás (Suire y Vicente, 2002). ${ }^{3}$

De frente a las tesis disciplinarias y sus respectivos enfoques complementarios desarrollados anteriormente, surge la inquietud de encontrar una perspectiva más cercana para posicionar el caso mexicano de la producción Web.

\subsection{Una posible inserción teórico-conceptual del caso de la polarización mexicana}

Adelantando algunos resultados del presente trabajo, destacamos que en el caso mexicano existe una fuerte centralización

\footnotetext{
${ }^{3}$ La primera lógica de aglomeración encuentra sentido en la explicación de los procesos de localización en el Silicon Valley (Saxenian, 1994 y 2000), y la segunda lógica se encuentra involucrada en la explicación del más reciente Silicone Sentier, en París (Suire y Vicente, 2002). En este último caso, la aglomeración se inició con un líder como Yahoo en 1998, seguido por Nomade en 1999; ambos generaron una imagen de viabilidad de la ubicación para las 300 empresas que en cascada se instalaron en el viejo barrio textil de Sentier (Vicente, 2002).
} 
territorial de la producción Web, en conjunto con una tendencia a la ampliación de las asimetrías entre las entidades federativas. Estos hechos observados, además de no encajar perfectamente en los marcos conceptuales dominantes, ${ }^{4}$ generan varias inquietudes (que se desarrollan más adelante) con relación a sus inserciones en la estructuración del discurso de la apropiación socioterritorial de las TIC.

En lo que toca a la www, la proliferación de un discurso alentador y generoso de la trascendencia de las TIC en los procesos de integración socioterritorial deja apenas entrever la bifurcación entre la lógica de producción de contenidos y las estrategias puestas en marcha para incrementar los niveles de su consumo (Zook, 2005). La diferencia entre las dos lógicas se encuentra plasmada, independientemente de la escala de análisis, en la polarización territorial de los actores, medios y habilidades que dan sustento a la producción de contenido en un entorno encaminado a expandir la cuenca de usuarios -al menos- en el segmento de la población económicamente solvente (Toudert, 2005). Así la Web es vista desde el enfoque del desarrollo de un mercado con características comparables a las demás actividades económicas y, por lo tanto, con un modesto potencial para despertar grandes inquietudes conceptuales. Sin embargo, nos encontramos lejos de tanta sencillez: el universo de temas e intereses de la www son moldeados en una reflexión plural, con mecanismos de apropiación de tecnologías y la estructuración de estilos de vida que, en conjunto, parecen dibujar un entorno multidimensional en la agitación del discurso de la sociedad de la información (Toudert y Buzai, 2004).

El discurso de la sociedad de la información ${ }^{5}$ toma posición en medio de un espectro complejo de significados y alcances otorgados a los contenidos de las redes públicas, y éstos no necesariamente son identificados como bienes y servicios mercantiles (Cornu et al., 2005). Como bien lo expresa Castells (2001), vivimos con agrado o por fuerza dentro de la galaxia Internet en medio de una sociedad de red cuyos flujos son vistos, por otros

\footnotetext{
${ }^{4}$ Por conceptos dominantes aludimos a los desarrollos teóricos con más aceptación en el entorno académico. En este trabajo, hacemos también mención del "discurso dominante" para caracterizar una construcción discursiva promocionada por comentaristas y líderes de opinión con un respaldo mediático consecuente.

${ }^{5}$ Nuestro acercamiento al concepto de la sociedad de la información va más bien encaminado a buscar un sentido a la polarización de la producción de contenidos vis a vis del discurso que envuelve el mismo concepto. Así, aunque compartimos la idea de una reflexión más centrada sobre los contenidos, el objetivo del trabajo no es indagar un modelo ideal de sociedad de la información.
} 
autores, como sinónimos de "información” y "comunicación”, una nueva forma de "aprendizaje colectivo" y producción de un "conocimiento accesible", la emergencia de una "inteligencia colectiva" como alternativa a las "jerarquías del conocimiento". ${ }^{6}$ En esos contextos, además de las características y finalidades del suministro de un servicio común, se consideran las interacciones y externalidades de la misma red (y a veces fuera de ella) como componentes fundamentales de la relación socioterritorial de los contenidos. De hecho, la relación entre contenidos y usuarios es una cuestión de escala que va incrementando los desacuerdos y contradicciones conforme se acerca a los órdenes territoriales básicos, los espacios naturales de dos dimensiones de alto significado social, económico y cultural como son: la información y la comunicación. Estas últimas son temáticas abordadas por el discurso dominante de la sociedad de la información desde un perspectiva general y un enfoque territorial global, desvinculándolas, en gran medida, de sus contextos inmediatos de generación (Toudert, 2005).

En el marco del discurso dominante, la colisión técnico-conceptual entre el incremento del número de usuarios, la participación en la producción de la www, la apropiación de las TIC y el nivel de integración a la sociedad de la información al posicionarse en un estrato global, conlleva a un "rompecabezas" teórico sin solución. De hecho, como lo advierte Wolton (2000), la tentativa de ubicar la información y la comunicación fuera de los alcances de la teoría social es poco convincente; en efecto, se estaría buscando insinuar un posicionamiento casual al despojarlas de relaciones sociales con los individuos, la democracia, la economía y la tecnología. Lo anterior nos lleva a considerar la inserción local y regional no como espacios neutrales de tránsito y/o de caída de los flujos de contenido, sino más bien como territorios potenciales estructurados por las redes y sus lógicas de construcción (Dupuy, 1991 y 1993). Sin embargo, aunque este tipo de inserción territorial aparezca como un supuesto viable y

\footnotetext{
${ }^{6}$ Expresiones que dan forma y contenido a un discurso multidimensional, complejo y ambiguo orientado a destacar, explicar y difundir los significados y alcances de la sociedad de la información. En la conceptuación de "inteligencia colectiva" de Lévy (1997, 1998), la ambigüedad de la visión mística de la red y sus contenidos es vista como un signo característico de la posmodernidad y su corolario: la libertad de interpretación. Sin embargo, desde una perspectiva "formal", en Souyri (2005) se afirma que la desmitificación del discurso de la sociedad de la información es una condición necesaria para la construcción de un marco conceptual con capacidad de mejorar la comprensión y la acción.
} 
de interés epistemológico razonable, se toma en cuenta que lejos de ser consensual, su uso es aun menos frecuente.

A pasar que las redes, como lo mencionan Bakis (1993b) y Brunet (2001), traducen las jerarquías territoriales existentes y a veces amplían sus márgenes, la rehabilitación del segundo término de la expresión global-local adquiere un significado estratégico para la reflexión y la acción; lo contrario sería quizá aceptar de antemano el veredicto implacable de la disolución de la iniciativa local y regional.

En lo que toca al desarrollo de las TIC, ante el desvanecimiento del recurrente mito de su distribución territorial equitativa, en proporción con la aparente incapacidad de los territorios "atrasados" para alcanzar a los más "adelantados”, se impone encontrar una alternativa al llamado de "solidaridad digital" de algunos países en proceso de desarrollo durante el Encuentro Mundial para la Sociedad de la Información (wsis) realizado en Génova, en el año 2003. De hecho, como comentan Eveno (1999) y Bertrand (2001), ni las decepciones ni los fracasos lograron matizar el atractivo poder de seducción de las TIC en los espacios locales y sus actores políticos. La creencia en la necesidad de la interconexión local a las redes como una condición sine qua non de la integración a la economía global (Graham y Marvin, 2001), nos manda de regreso a una de las profecías fallidas de McLuhan: "The meduim is the message". En este sentido, las TIC, antídoto a la desmovilización territorial de los actores de una geografía sin fricción y el voluntarismo que impregna algunos segmentos del discurso de la "brecha digital", son las caras de una misma moneda vista desde el ángulo de la obsesión de traducir los medios por sus finalidades.

La finalidad del desarrollo puede verse según lo que comenta Veltz (2002), como un producto de una coevolución compleja, un ecosistema relacional entre las estrategias corporativas y los actores territoriales, que pueden respaldar una cooperación menos sujetada a la distancia. Sin embargo, la autonomía en la construcción de una atracción local y regional depende de la capacidad de tomar decisiones en materia de políticas públicas encaminadas hacia la creación y consolidación del capital social, y estas prerrogativas son generalmente concentradas en los poderes de las jerarquías territoriales superiores (Puel y Vidal, 2003).

Por lo anterior, la interpenetración de las esferas de decisión en la ecuación global-local deja apenas entrever otra ambigüedad que toma posición en la pertinencia de las escalas de análisis de la producción Web. Además de la confusión sobre lo que cada 
quien entiende por local y global, carecemos de un conocimiento satisfactorio sobre el impacto de la segmentación territorial de la producción de contenidos y el interés que éstos generan en los usuarios locales y regionales. O sea, qué tanto la oferta de contenidos puede ser independiente de las especificidades y antagonismos de la demanda local, y qué tanto se puede lograr lo anterior ubicando la oferta fuera del contexto territorial del usuario. La articulación local en este sentido lejos de asemejarse a una acción automática y neutral, toma posición en la disponibilidad de medios y habilidades adquiridas, en cierta medida, en los procesos de aprendizaje y la práctica de la generación de contenidos similares (Toudert y Buzai, 2004). Todo lo dicho, en el marco del discurso de la sociedad de la información, nos lleva a replantear la posición de las lógicas de aglomeración local de cara a la polarización que prevalece en los procesos de producción de contenidos Web.

El hecho mismo de la polarización pone sobre la mesa de discusión un argumento suficiente para descartar la perspectiva de la aglomeración sustentada en la disolución de la fricción espacial y, por lo tanto, es más viable un acercamiento desde la dependencia territorial de los procesos de localización. Del mismo modo, es necesario subrayar, una vez más, que la tesis de aglomeración basada estrictamente en la trascendencia de las interacciones económicas presenta una explicación incompleta de los acontecimientos observados. En este caso de estudio, la polarización de los indicadores de la actividad económica no se asemeja ni se compara de manera lineal con la fuerte centralización de la producción Web en México. Como posible línea de investigación a futuro, se plantea tomar en cuenta las externalidades de tipo informativo y las estrategias organizacionales que parecen estructurar un marco explicativo complementario a lo vinculado con las interacciones económicas.

En materia de desarrollo de la www, a pesar de que la polarización a escala de los diferentes países fue puesta en evidencia a partir de la década de los noventa el análisis de los entornos intranacionales se enfocó, en gran medida, a un grupo selecto de países desarrollados, con una sobreexposición en el caso estadounidense (véanse los trabajos de Moss y Townsend, 1997 y 2000; Kolko, 2000, y Zook, 2000 y 2001). Lo anterior, además de conllevar a una marginalización analítica de los contextos inherentes a los países emergentes, deja entrever una posible interacción sesgada entre el arbitraje empírico y la búsqueda de una teoría unificada para el desarrollo territorial de la Web (Toudert, 2003). Los resultados expuestos en este trabajo nos 
llevan a sospechar de la existencia de diferencias pertinentes entre los dos grupos de países y, por lo tanto, nos preguntamos si la evidencia es suficiente para abrir una exploración en los países emergentes.

La prevalencia de un entusiasmo impregnado de una cierta ingenuidad en un entorno de relativa debilidad de la geografía y sus disciplinas para lograr una construcción epistemológica de las TIC, conduce a una carencia de la información local y regional (Bakis, 1993a; Hillis, 1999; Toudert, 2000). La concentración del análisis en una escala de países se traduce, en gran medida, en la centralización del sistema de gobierno de la Internet y en su incapacidad para encajar en un marco operativo local y regional eficiente ${ }^{7}$ (Toudert y Buzai, 2004; Damilaville, 2005). En medio de tantas deficiencias conceptuales y operativas en la generación de datos e información, el desarrollo instrumental para apoyar el presente trabajo constituye un logro inicial y, sin lugar a dudas, perfectible.

\section{Procedimientos y mecánica de generación de datos}

En el presente trabajo se hizo un manejo de datos generados según fuentes y procedimientos diferentes. En lo que toca a la información de dominios Web, hasta donde tenemos conocimiento, es la primera vez que se generan estos datos en el contexto territorial interno de un país emergente y, por lo tanto, esperamos contar con la indulgencia de nuestros lectores con relación a nociones que se encuentran todavía en las miras de la investigación.

\subsection{La información generada en el marco de las encuestas de dominios}

La información de dominios se generó en el marco de dos encuestas nacionales "Cibermex" levantadas bajo nuestra responsabilidad durante el 2000 y $2004 .{ }^{8}$ La encuesta del 2000 se llevó a cabo

\footnotetext{
${ }^{7}$ La cooperativa alemana DENIC-eG es la única estructura con visión territorial local; fue creada por corporaciones e instituciones en 1996 con la finalidad de autorregular el desarrollo de la Internet y ofertar una información de dominios desagregada por diferentes divisiones territoriales.

${ }^{8}$ Encuestas realizadas gracias al apoyo financiero recibido en el marco de las convocatorias internas de apoyo a proyectos de investigación de la Universidad Autónoma de Baja California. Estas encuestas son identificadas con los nombres genéricos de Cibermex 2000 y 2004, y vienen a consolidar el proyecto permanente "Ciberespacio, territorios y territorialidad en México”.
} 
con la finalidad de evaluar la validez y la eficiencia del procedimiento metodológico; su campo de exploración fue restringido al conteo y ubicación territorial de dominios. La encuesta del 2004 es una réplica de la iniciativa del 2000, pero en ella se extendió el campo de búsqueda a un conjunto más amplio de variables.

Ambas encuestas se llevaron a cabo en estricta conformidad con los marcos metodológicos e instrumentales divulgados detalladamente en Toudert y Buzai (2004). De manera general, el proceso muestral se desarrolló en un padrón de dominios actualizado automáticamente con el apoyo de un algoritmo apuntando hacia los buscadores con mayor propensión a alojar sitios bajo la bandera .Mx. Con la restricción anterior se asumió que sólo los dominios .MX constituyen el universo producido en México, cuando en la realidad se dan los casos de sitios mexicanos registrados con dominios de otros países y viceversa. ${ }^{9}$ Sin embargo, a la luz de la poca evidencia existente, se estima que los sitios registrados fuera de sus dominios "nacionales" no alteran las tendencias observadas (Toudert y Buzai, 2004). ${ }^{10}$

El proceso muestral propiamente dicho, se realizó con apoyo en el padrón de sitios, tomándose como universo los dominios registrados por el NIC-México. ${ }^{11}$ Para conformar un marco muestral caracterizado por un error proporcional absoluto permitido de $2 \%$ y un nivel de confiabilidad de $95 \%$ en los resultados inferidos, se seleccionó aleatoriamente una muestra estratificada proporcional aplicando una frecuencia de sondeo de $1 / 15$ en el 2000 y de $1 / 50$ en 2004 . Una vez definida la muestra, se dio paso a la ubicación territorial de dominios en las bases de datos Whois

${ }^{9}$ Network Information Center-México (NIC-Mexico) es la organización encargada de la administración del código país del nombre de dominio (cc-TLD) .MX. De manera general, se le agrega a una dirección Web (URL) un nombre de dominio genérico (gTLD: com, net, org, gob, edu) y el cc-TLD del país de correspondencia (cc-TLD: MX, ES, AR). El sistema de gestión de las URL permite usar direcciones Web nominales en lugar del complicado Internet Protocol Adress (IP adress) de cuatro números entre 0 y 255.

${ }^{10}$ Para prevenir la especulación con los dominios, existe la práctica de registrarlos con varias modalidades de "cC-TLD" y "gTLD". En lo general, registrar un dominio principal con el "cc-TLD" del país de correspondencia facilita la transcripción exacta de la dirección por parte de los usuarios nacionales. Sesenta y cinco por ciento de las 500 empresas más importantes de México en el 2004 tuvieron una dirección principal con un "cc-TLD" .MX (Expansión, 2005); las demás son identificadas principalmente con los "gTLD", aun cuando presentan, en su gran mayoría, un dominio secundario con un "ccTLD”.MX.

${ }^{11}$ Para el desarrollo de las encuestas 2000 y 2004, se tomaron como universos totales las estadísticas del NIC-México para el 30 de abril del 2000 y 23 de agosto del 2004, fechas que corresponden a los periodos en que se efectuaron el proceso de muestreo y el levantamiento de cada encuesta. 
disponibles por medio de organizaciones como la InterNic. ${ }^{12}$ Lo anterior dio como resultado una relación de dominios por ubicación geográfica que permitió el cálculo de la densidad de dominios por cada mil habitantes con base en el dato poblacional del Censo General de Población y Vivienda 2000 y su proyección para el 2004.

\subsection{Generación y homogeneización del número de usuarios de Internet por estado}

La Asociación Mexicana de Internet (AMIPCI) realiza anualmente, desde el año 2000, por medio de la consultora Selet, la encuesta de "Hábitos del mercado en línea en México". A partir de ese año se incluyó una pregunta para ubicar a los usuarios de Internet en los estados de la República, publicándose los resultados sólo para las entidades con una participación pertinente en el número total de usuarios. ${ }^{13}$ Los datos generados, además de presentar falta de información para varios estados de la República, se caracterizaron también por cortes anuales (publicados de acuerdo con la lógica del ranking), conformando listas de estados parcialmente diferentes de un año a otro. Para hacer uso de estos datos de generación única en el país, tuvimos que realizar dos procesos de extrapolación de la información a las demás entidades federativas ausentes de las publicaciones de la AMIPCI.

En primer lugar se realizó para los estados presentes en la lista publicada anualmente por AMIPCI una proyección de la participación de usuarios por entidad que permitió lograr la homogenización de la información de usuarios para el 2000 y 2004. En un segundo paso se aplicó a las encuestas del 2000 y del 2004 un modelo de predicción lineal regresivo para la generación de la información faltante para algunas entidades federativas. El modelo logrado fue optimizado con un estudio exploratorio discri-

${ }^{12}$ La evidencia estadounidense de una correlación positiva entre el registro de ubicación de dominios y las direcciones de las organizaciones propietarias provino de la base de datos de las consultoras CorpTech (véase los trabajos de Zook, 2000 y 2001) y Maxmind. En el caso de esta última consultora, se sostiene un porcentaje de coincidencia de $78 \%$ en un radio de 25 millas para México. Sin embargo, coincidimos con Castells (2001) en afirmar que la mayoría de las discrepancias proviene más bien de la imprecisión de las bases de datos toponímicas utilizadas para ubicar los registros de dominios.

${ }^{13}$ Estas encuestas, por ser levantadas en portales en línea orientados a segmentos específicos de usuarios, dejan entrever un posible sesgo. A pesar de que la diversificación de estos portales (34 en el 2004) juega en favor de una mayor representatividad, tenemos algunas reservas con relación a la repartición de la muestra entre estos portales por la evidente falta de un conocimiento independiente sobre los perfiles de usuarios. 
minante que arrojó como variables independientes: a) porcentaje de dominios por estado de cara al total nacional; $b$ ) participación estatal en el producto interno bruto (PIB) nacional, y c) captación bancaria por habitante en el nivel estatal con relación al mismo concepto en el nivel nacional. ${ }^{14}$

Una vez determinada la participación de los usuarios de Internet en cada estado de la Republica para el 2000 y el 2004, se calculó el número absoluto de usuarios con base en el total nacional de usuarios estimado por las encuestas de la AMIPCI. Los demás cálculos e indicadores que implican densidades por número de habitantes se basaron en el dato poblacional del Censo General de Población y Vivienda 2000 y su proyección para el 2004.

\section{Resultados y caracterización de la producción Web en México}

Para lograr una caracterización coherente de la producción de la WWW en el nivel de las entidades federativas, los resultados de este trabajo se presentan desde tres perspectivas encaminadas a facilitar, en la última sección, la discusión comparativa con otros contextos territoriales. De acuerdo con esta lógica, en el inicio se enfoca la contribución de los estados a la producción nacional de la Web, seguida por la exploración estructural de la dispersión territorial en el entorno de las contribuciones estatales, y, para finalizar, se pone de relieve lo que consideramos, desde nuestra perspectiva, como rasgos característicos de la especialización estatal.

\subsection{Una aplastante polarización territorial de la estructura productiva de la Web}

En su conjunto, la contribución de las 15 primeras (top-15) entidades federativas productoras de la Web se mantuvo durante el periodo 2000-2004 casi constante en una cifra aproximada de 94\% del total de los dominios producidos en México. Durante el mismo tiempo, la producción Web fue muy marginal en los 17 estados restantes, que registraron individualmente una contribución promedio del orden de $0.35 \%$ a la www nacional.

\footnotetext{
${ }^{14}$ La variable "b" para el 2000 fue tomada del sistema de consulta Web del Banco de Información Económica del Instituto Nacional de Estadística, Geografía e Informática (INEGI); para el 2004, la participación estatal en el PIB nacional fue proyectada con datos de la misma fuente. La variable "c" fue tomada de las publicaciones de Banamex-Citigroup (2001 y 2004).
} 
La producción nacional de la Web fue encabezada por el Distrito Federal (DF), Nuevo León y Jalisco con una participación que presentó un incremento entre 2000 y 2004 de 73 a $75 \%$ del total de los dominios, destacando en el mismo periodo una franca dominación del DF, con una contribución que pasó de 53 a $55 \%$ del total nacional (véase gráfica I).

La dinámica 2000-2004 de los estados productores de la Web fue marcada en el 2004 por la salida del top-15 de los estados de Sonora, Sinaloa y Coahuila y la entrada de Quintana Roo, Yucatán y Guerrero. Sin embargo, hay que aclarar que las tres entidades que ingresaron al top-15 durante el 2004 lo lograron con una participación de sólo 4\%, que puede tradurcirse en una dinámica marginal que se presenta en el nivel bajo del top-15.

Desde una perspectiva estandarizada con la población, la contribución en el 2004 de las entidades federativas medida con indicadores de penetración social de Internet y de especialización en la producción de la Web destaca un alto distanciamiento entre el DF y el promedio nacional. En este sentido, el DF presentó una participación 18 veces más alta en la producción de dominios Web, 11 veces más usuarios de la Internet, 2.5 veces más

\section{Gráfica I}

Dinámica de los 15 primeros estados productores de dominios Web entre 2000-2004

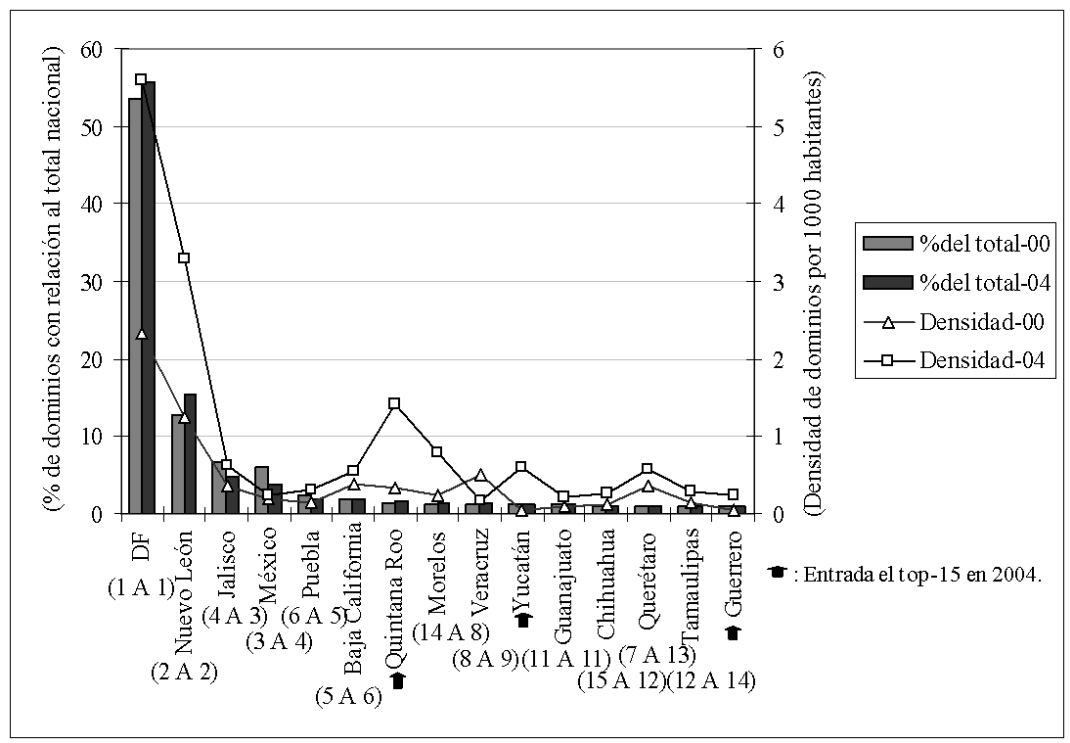

Fuente: Véase la sección "Procedimientos y mecánica de generación de datos". 
dominios Web por cada cien usuarios de Internet y, finalmente, 10 veces más dominios por cada mil habitantes. A pesar del matiz que introduce la estandarización con la población en los dos últimos indicadores, salvo el estado de Nuevo León, que presentó dos veces más dominios Web por cada cien usuarios de Internet que el DF, el dominio del DF en los demás casos es aplastante.

\subsection{Estancamiento de las asimetrías entre entidades federativas}

De frente a la fuerte polarización que pareció caracterizar a la producción Web en México entre el 2000 y el 2004, el análisis de la dispersión de las contribuciones estatales nos permite apreciar la tendencia evolutiva de la iniquidad en las contribuciones que consolida a final de cuentas la centralización.

La dinámica de la dispersión en la contribución de los estados a la producción de la Web nacional medida por la evolución del coeficiente de variación (CV) ${ }^{15}$ interestatal entre 2000 y 2004 re-

\section{Gráfica II}

Evolución interestatal de algunas variables de penetración de la Internet y la Web entre 2000 y 2004

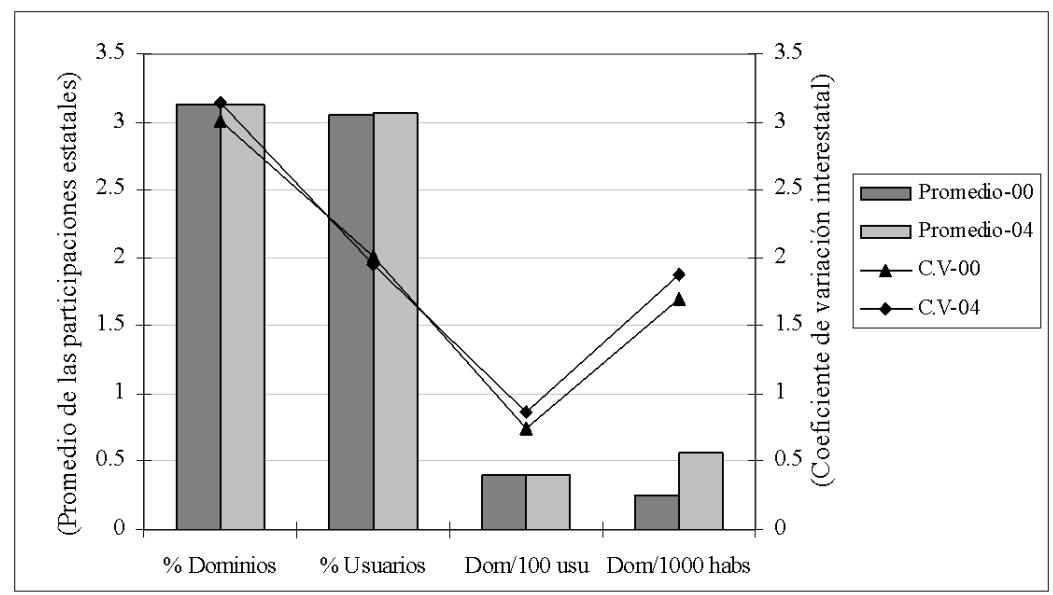

Fuente: Véase la sección "Procedimientos y mecánica de generación de datos".

${ }^{15}$ El coeficiente de variación (cv) es el resultado de la relación entre la desviación típica y el promedio. El cv es una medición sencilla y sin unidad que permite determinar el grado de dispersión del indicador de interés en las entidades de análisis. De este modo, un cv bajo corresponde a un menor grado de concentración, lo que significa una mayor equidad de la repartición y viceversa. 
gistró un ligero incremento, pasando de 3 a 3.13, lo que deja entrever una relativo estancamiento de las diferencias observadas (véase gráfica II).

De manera general, se puede observar que la concentración en la contribución de las entidades federativas es más baja en el caso de los indicadores estandarizados con la población respecto a los demás indicadores absolutos. En el mismo orden de ideas, durante el 2004 la concentración de la contribución interestatal en el número de dominios por cada mil habitantes fue dos veces más elevada que en el número de dominios por cada cien usuarios de Internet. Esta situación parece indicar que a pesar de haberse duplicado el promedio interestatal de dominios por cada mil habitantes entre el 2000 y el 2004, hay más equidad en la contribución de los estados a la producción de la Web entre los usuarios de la Internet que entre la población en general (véase gráfica II). El estancamiento temporal de la dispersión interestatal entre usuarios y población en general, se traduce en las diferencias, a veces muy altas, entre los dos universos y, en cierta medida, en un fuerte desfase en las dinámicas del crecimiento de los usuarios de Internet respecto al crecimiento poblacional. ${ }^{16}$

\subsection{Fortalecimiento de un eje productivo bicéfalo entre el DF y Nuevo León}

Enfocando el análisis a los usuarios de la Internet, que son finalmente los beneficiarios directos de la producción de la Web, el cociente de consumo de la Web (CCW), ${ }^{17}$ introducido por primera vez por Zook (2001), constituye un indicador de interés para apreciar la evolución de los comportamientos estatales respecto el promedio del desempeño nacional.

Las entidades federativas que mostraron un CCW más alto que el promedio nacional durante el 2000 sumaron un total de siete; en orden decreciente: Morelos, DF, Querétaro, Puebla, Nuevo León, Tlaxcala y Jalisco. Durante el 2004 se registro un cambio significativo; las entidades con mayor desempeño que el prome-

${ }^{16} \mathrm{Al}$ cierre del 2004, el universo de usuarios de Internet en México alcanzó $14^{\prime} 901,687$, con una tasa de crecimiento anual, en baja de 35.4\% entre 2001 y 2002 a $21.6 \%$ entre 2003 y 2004, y se estima para el 2004-2005 una tasa aún más baja, del orden de $14 \%$ (AMIPCI, 2004).

${ }^{17} \mathrm{CCW}=$ (Número de dominios Web del estado i / Número de usuarios del estado i) / (Número de dominios nacionales /número de usuarios nacionales). Cuando el ccw de un estado es superior a 1, el consumo es superior al promedio nacional, y cuando es inferior a 1 , el consumo estatal es inferior el promedio nacional. 
dio nacional fueron seis, conformadas en orden decreciente por: Nuevo León, DF, Quintana Roo, Sinaloa, Baja California Sur y Yucatán (véase lámina I).

La dinámica entre el 2000 y el 2004 del CCW más alto que el promedio nacional destaca la pérdida de la continuidad territorial en la región central del país como producto de un bajo desempeño de las entidades que conforman la cuenca de usuarios potenciales: Jalisco y los estados periféricos del DF. A pesar de una distribución territorial que parece indicar signos de un relajamiento de la polarización en el 2004, la misma dinámica deja entrever una franca consolidación del liderazgo territorial de Nuevo León y el DF acompañada de un desplome del CCW en la entidad de Jalisco. Lo anterior nos lleva a reconsiderar la pertinencia de seguir exhibiendo el "Triángulo de Oro"18 como el defecto estructural del desarrollo de las TIC en México, cuando todo parece indicar un fortalecimiento de la polarización en un eje de desarrollo bicéfalo conformado por Nuevo León y el DF.

La emergencia de nuevas entidades en el 2004, como Quintana Roo, Sinaloa, Baja California Sur y Yucatán, es más bien el resultado de un crecimiento de la participación de esos estados en la producción de la Web en un entorno caracterizado por un número de usuarios relativamente bajo. Sin embargo, hay que aclarar que la participación en la producción nacional de la Web de esas mismas entidades es drásticamente reducida y, por lo tanto, una producción marginal tendrá un impacto pertinente en el CCW. Dicho de otro modo, bajo una cierta masa crítica de dominios Web y usuarios de Internet, el distanciamiento de los componentes del CCW entre entidades es tal que el indicador pierde coherencia y significado.

\section{Discusión y contextualización comparativa de los rasgos observados}

La Web en México se inició formalmente en 1994 con 45 dominios, llegando a alcanzar durante el mes de marzo del 2005 la cifra de 116,616. En su gran dimensión, la Web mexicana se encuentra capitalizada internamente y estructurada alrededor de pequeños jugadores (Toudert, 2005). Entre el 2000 y el 2004, los dominios en México tuvieron una tasa de crecimiento promedio anual de $16.58 \%$, y la densidad de dominios por cada mil habi-

\footnotetext{
${ }^{18}$ Una expresión muy difundida entre los profesionales, analistas y comentaristas del desarrollo de las TIC en México para destacar la polarización de los medios y oportunidades en un triangulo conformado por tres entidades: el DF, Jalisco y Nuevo León.
} 

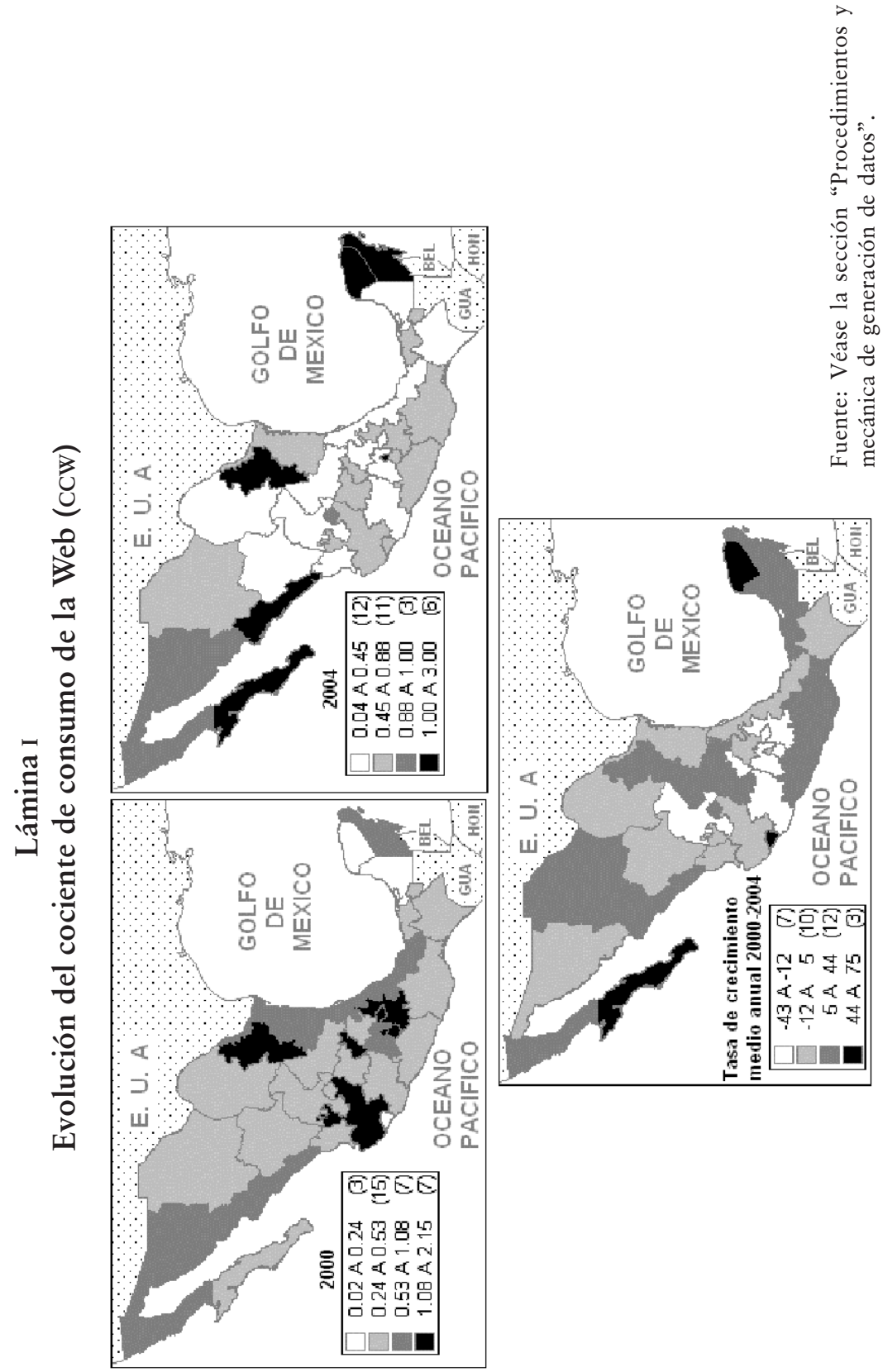
tantes pasó de 0.54 en el 2000 a 0.90 en el 2004, marcando un crecimiento promedio anual de $15.04 \% .{ }^{19}$

En una comparación de la densidad en México de dominios por cada mil habitantes con la de los países de la Organización para la Cooperación y el Desarrollo Económicos (OCDE), en el 2002, la cifra nacional se ubica en alrededor de 31 puntos porcentuales por debajo del promedio de la OCDE, y en 37 puntos por debajo del de la Unión Europea. ${ }^{20}$ Con el crecimiento registrado entre el 2000 y el 2004 de la densidad de dominios en México, le tomaría a nuestro país 15 años alcanzar la densidad promedio de la OCDE en el 2002 (OCDE, 2003).

Entre otros comentarios que se pueden verter respecto al alto desnivel observado entre México y los países desarrollados, rescatamos el hecho de un evidente potencial de crecimiento que se desprende del mismo diferencial, que tendrá que reducirse en cualquier escenario optimista. Sin embargo, nos sumamos a Castells (2001) preguntándonos por qué los sitios del mundo virtual son puestos en marcha desde pocas ubicaciones y a partir de un enfoque local; podemos seguir preguntándonos también si la polarización territorial de la Web en México es compatible con el propósito de aminorar la brecha con los países desarrollados. En un contexto de falta de datos para contestar de manera satisfactoria a todas las dimensiones de las preguntas anteriores, un acercamiento inicial comparativo entre México y los países desarrollados puede sustentar un interés para profundizar en un futuro la investigación en este sentido.

La caracterización en la sección anterior de la producción Web en el nivel de las entidades federativas deja entrever particularismos y divergencias del conocimiento publicado con relación a la misma producción en algunos países desarrollados. Sin embargo, como se ha venido afirmando desde el inicio, es poca la literatura con objetivos coincidentes, por ello, tanto nuestra fuente comparativa en lo que toca a los datos globales y estadounidenses fue la generada por Zook (2000 y 2001), así como la información del contexto alemán generada por la cooperativa DENIC-eG. ${ }^{21}$

${ }^{19}$ Datos generados con información del Nic-México y con la proyección de la población a partir del Censo General de Población y Vivienda 2000.

${ }^{20}$ Cálculos realizados con datos publicados por la OCDE en el 2003. Conviene mencionar que la densidad de dominios reportada por la ocDE para México en el 2000 es 0.3 más baja que la calculada con datos del NIC-México y Censo General de Población y Vivienda 2000; el diferencial representa casi 30,000 dominios, o sea, 55\% de los dominios totales registrados por el NIC-México hasta el 31 de agosto del 2000.

${ }^{21}$ Hasta donde tenemos conocimiento, existen sólo cuatro referentes comparativos con nuestro trabajo. En el área estadounidense, la primera fuente proviene de los 


\subsection{La polarización como denominador común, pero exageradamente alta en México}

La producción de la Web en México no escapa a las grandes lógicas de polarización existentes en varios países desarrollados; sin embargo, los niveles de centralización en nuestro país son exageradamente elevados. En efecto, cuando en el 2000 la más alta participación de la producción de la Web estadounidense fue alcanzada por el estado de California con 21\% (Zook, 2000 y 2001), y en Alemania por la Renania del Norte con $24.18 \%,{ }^{22}$ en México, el DF encabezó las entidades federativas con una contribución de 53\%. Durante el mismo año, el top-15 de los estados productores de la Web estadounidense registro una participación de $67 \%$, frente a una contribución de $94 \%$ alcanzada por el top-15 en México. En el mismo orden comparativo, el Reino Unido presentó en 1999 la producción nacional más polarizada de los países europeos con una participación de 29\% en Londres (Zook, 2000 y 2001). mientras que en Alemania se registró la participación menos centralizada del continente: en conjunto, Berlín, Munich y Hamburgo contribuyeron con sólo 13.2\% a la producción nacional de la Web. ${ }^{23}$

Enfocando la disimetría de las contribuciones estatales, la situación nacional es también particular: cuando en México el coeficiente de variación (CV) de las producciones estatales de la Web alcanzó una cifra de 3 en el año 2000, el CV estatal estadounidense fue de 1.7, y el de los 16 länder alemanes apenas alcanzó 1.08, es decir, la concentración de las participaciones estatales en México es casi dos veces mas alta que en Estados Unidos y tres veces más que en Alemania. ${ }^{24}$

trabajos de Moss y Townsend entre 1997-1998 que se apoyan en los datos de la consultora Imperative!; la segunda consta en lo publicado por Zook entre 2000-2001, incluyendo un comparativo de 2,500 ciudades del mundo; la tercera fuente proviene de estadísticas alemanas divulgadas periódicamente por la cooperativa DENIC-eG, que dio sustento a la publicación de Krymalowski (2000), y la última fuente proviene de la investigación Dodge y Shiode (2000), enfocada en el Reino Unido. Para llevar a cabo el análisis comparativo se descartaron las fuentes basadas en datos anteriores a las fechas de nuestro trabajo (Moss-Townsend, 1997; Krymalowski, 2000 y Dodge y Shiode, 2000).

${ }^{22}$ Datos de DENIC-eG, 2000 y 2004.

${ }^{23}$ Cálculo con datos de DENIC-eg, 2000 y 2004.

${ }^{24}$ Cálculo para Estados Unidos realizado con datos del conteo de dominios provenientes de la base de datos del Dr. Matthew Zook, de la Universidad de California, Berkeley. En el caso alemán se usaron datos de DENIC-eG. 
En el marco de la información comparativa disponible, la centralización territorial de la producción de la Web en México deja entrever un rostro distinto al de los países desarrollados. El análisis comparativo parece arrojar esquemas de localización que no reflejan linealmente y en la misma medida la tradicional atractividad de los centros de gravedad de la actividad económica; en los países desarrollados parece predominar una polarización relativamente extensiva, y en México sobresale una lógica de centralización francamente intensiva. Lo anterior, con el paso del tiempo, nos lleva a preguntar si hay indicios de una mayor difusión e integración socioterritorial en el marco de la lógica actual del desarrollo nacional de la Web.

\subsection{Una evolución divergente de la polarización mexicana respecto a los países desarrollados}

En lo relativo a la información internacional disponible, el dato comparativo más pertinente en la dinámica evolutiva de México, Estados Unidos y Alemania es la variación temporal de las participaciones territoriales en las respectivas producciones nacionales de la Web. Así mientras la participación del top-15 de las entidades estadounidense pasó de $74 \%$ en 1998 a $58 \%$ en el $2001,,^{25}$ y entre el 2000 y el 2004 el top-15 de ciudades y regiones alemanas bajó su participación en una proporción modesta de 26.5 a 25.7\%, en México, durante el mismo tiempo, la contribución del top-15 a la producción de la Web nacional se mantuvo casi constante, en alrededor de 94\%. En lo que toca a las entidades con mayor contribución, la participación del estado de California en la producción estadounidense disminuyó de $21.5 \%$ en 1998 a 17\% en el 2001, y la participación entre 2000 y 2004 de Berlín, Hamburgo y Munich en la producción alemana bajó de $13.24 \%$ a $12.47 \%,{ }^{26}$ por su parte, durante ese mismo período la participación del DF siguió subiendo de 53 a 55\%. Lo anterior parece indicar que con el paso del tiempo, mientras en Estados Unidos y Alemania ha habido una difusión de la participación territorial a la producción nacional, en México nos encontramos de cara a una creciente concentración de la Web. Sin embargo, aunque el comparativo entre Estados Unidos y Alemania parece insinuar que a mayor polarización corresponde una mayor difusión en el tiempo, en el caso mexicano no se ve del todo esta

${ }^{25}$ Cálculo a partir de la base de datos del Dr. Mattew Zook.

${ }^{26}$ Véase nota 24. 
tendencia. De hecho, entre 2000 y 2004 en México se registró un aumento en el cv de la participación de los estados, que pasó de 3 a 3.13, traduciéndose en un crecimiento de la iniquidad en la contribución de las entidades federativas, cuando en Alemania la tendencia fue lograr una mayor equidad en la participación territorial de sus 16 länder (el CV pasó de 1.08 a 1.06) y hasta de sus 440 ciudades y regiones más importantes (el cv bajó de 1.98 a 1.83$).{ }^{27}$

Además de las particularidades de la concentración que caracteriza al DF, se registró en la misma entidad un indicador de especialización en la producción de la Web relativamente bajo respecto a varias capitales y ciudades de los países desarrollados. En efecto, cuando en el 2000 hubo en el DF 2.33 dominios por cada mil habitantes, en 1999 Los Ángeles alcanzó 21.7 Nueva York, 16.8; Vancouver, 10.9; Londres, 8.6; París, 2.5; Berlín, 20.2; Hamburgo, 20.2; Munich, 50.9, y Tokio, 1.3 (Zook, 2000 y 2001 y Krymalowski, 2000). Siendo la densidad de dominios por cada mil habitantes muy baja en el DF, lo anterior evidencia una gran capacidad de desarrollo que se puede apreciar en el crecimiento medio anual del $24.4 \%$ entre 2000-2004, de frente a $20.12 \%$ registrado en el land de Berlín durante el mismo periodo. ${ }^{28}$ En el mismo orden de ideas, el crecimiento alcanzado en la densidad por el DF es nueve puntos porcentuales más alto que el incremento nacional y 11 puntos porcentuales por debajo del incremento promedio registrado entre 2000 y 2002 por los países de la OCDE.

De todo lo anterior sobresale en México una polarización territorial con un perfil que destaca a futuro un gran potencial de crecimiento de la producción Web, alimentando a su vez un padrón de concentración acaparado en su más alta proporción por el DF. Desde esta perspectiva, el desarrollo de la Web adquiere un significado y una apreciación de escala en donde los indicadores nacionales son sustentados en un desempeño territorial altamente diferenciado. La oportunidad del desarrollo nacional de la Web se vuelve casi sinónimo de la capacidad de su crecimiento en el DF, dejando entrever, por lo tanto, serios riesgos de fortalecimiento de la polarización existente.

Más allá de lo observado en México, la búsqueda de una hipotética unidad en el discurso de la sociedad de la información

${ }^{27}$ Cálculo realizado con datos de DENIC-eG, 2000 y 2004.

${ }^{28}$ Entre 2000-2004, el número de dominios por cada mil habitantes pasó en el DF de 2.33 a 5.58, mientras en el land de Berlín hubo un incremento de 61.98 a 129.06. 
nos lleva a preguntar si nuestro contexto nacional es aislado $\mathrm{u}$ obedece a una lógica compartida con los demás países emergentes. Dar respuesta a esta pregunta en un entorno informativo más propicio de lo disponible en la actualidad, permitirá quizá reposicionar la apropiación socioterritorial de las TIC en el marco de la divergencia de lógicas de aglomeración entre países desarrollados y emergentes.

En este sentido, la naturaleza del desnivel con los países desarrollados nos invita a considerar la intervención de diferencias pertinentes en la apropiación socioterritorial de las TIC y su posible traducción en padrones distintos de aglomeración productiva. Lo anterior abre la posibilidad de plantear alternativas y conducir políticas encaminadas a suavizar los excesos que pueden caracterizar a la producción Web en países como el nuestro.

\subsection{La conexión entre polarización organizacional y centralización de contenidos}

La polarización de la producción Web en México no parece estar sujeta totalmente a una lógica de estructuración territorial del desarrollo económico; al ser así, observaremos una relación clara entre el ranking de la contribución estatal a la producción Web y los indicadores de la actividad económica, y quizá también los demás indicadores del desarrollo social. ${ }^{29}$ Por un lado, la actividad económica no parece explicar por sí misma la fuerte centralización de la producción Web en el DF, y, por el otro, salvo el caso de Nuevo León, la contribución de las demás entidades federativas no manifiesta sus pesos respectivos en la economía nacional.

Los empiristas de primera generación coincidieron en explicar la centralización de la producción Web estadounidense por medio del nivel de conexión metropolitana a lógicas y jerarquías

${ }^{29}$ Es común en ciertas organizaciones internacionales (Organización para la Cooperación y el Desarrollo Económico: ocDE y Unión Internacional de Telecomunicaciones: UIT) insinuar a escala de países una cierta relación causal entre los niveles de apropiación de las TIC y los indicadores del desarrollo socioeconómico. Lo anterior influye en la construcción de un discurso operativo que pretende lograr uno de los dos objetivos proporcionando las condiciones del otro. En esta parte del trabajo no se busca discutir la validez de estas afirmaciones y supuestos, lo que pretendemos es atraer la atención sobre el pertinente diferencial existente entre el grado de polarización de la Web en México de frente a la centralización de las actividades económicas señalada por una extensa literatura sobre el tema. Asimismo, observamos que el ranking de las entidades federativas de la gráfica I no refleja una correspondencia significativa con indicadores comunes como el PIB per cápita, el número de unidades económicas, indicadores del desarrollo humano, la teledensidad... etcétera. 
de acceso a la información económica (Moss y Townsend, 1997 y 2000; Dodge y Shiode, 2000). A la dimensión anterior, Zook (2001) añade la disponibilidad en algunas metrópolis (y no en otras) de condiciones organizacionales y estructurales encaminadas a centralizar la innovación tecnológica y la inversión del capital-riesgo, que en conjunto concurren al fortalecimiento de la polarización de contenidos.

Estos entornos parecen caracterizar a una Web compuesta de jugadores con un cierto nivel de sofisticación en materia de innovación y una pertinente estructuración de sus planes de negocio en la modalidad virtual (o en línea). Sin embargo, resulta quizá necesario reconocer que pocos actores de la Web están involucrados en acciones de liderazgo en la innovación tecnológica y no todos son financiados por medio del capital-riesgo. En el caso de México, durante el 2004 sólo 12\% de los dominios registrados fueron originados por organizaciones con planes de negocio posicionados exclusivamente en línea, y la mitad de ellos se ubicaron en el $\mathrm{DF}^{30}$ En el mismo orden de ideas, los dominios nacionales provenientes de organizaciones claramente posicionadas en informática, Internet y telecomunicaciones, apenas alcanzan 13.54\% (con un poco más de la mitad localizados en el $\mathrm{DF})$; los demás son principalmente el producto de un entorno comercial, de prestación de servicios y de gobierno (Encuesta Nacional Cibermex, 2004). La Web mexicana aparece, entonces, sustentada en su más extensa dimensión por actividades tradicionales y presenciales complementadas en la modalidad virtual con diversas lógicas de optimización, promoción y diversificación. En este sentido, la producción de contenidos Web en México presenta entornos estructurales conectados a contextos socioterritoriales favorables, unos más que otros, a la apropiación de las TIC.

La reciente incursión del empirismo de segunda generación en el estudio del caso estadounidense arroja evidencias de contextos de apropiación de las TIC segmentados por diferentes lógicas organizacionales en el nivel de los sectores, ramas y clusters, a veces de manera independiente de la ubicación en territorios dominantes (Forman et al., 2005; Greenstein, 2006). Otras evidencias ponen de relieve el papel de las externalidades de tipo

\footnotetext{
${ }^{30}$ Del total nacional de los dominios con planes de negocio estrictamente en línea, $52 \%$ se especializan en las categorías de Internet y computación, $18 \%$ en comercio, $11 \%$ en portales verticales, $5 \%$ en sociedad y cultura, y $6 \%$ en vida local y regional (Encuesta Nacional Cibermex, 2004).
} 
informativo en los procesos de ubicación en contextos de menor jerarquía territorial y/o de baja articulación entre actores (Capello y Nijkamp, 1995; Zimmermann, 2001; Suire y Vicente, 2002).

Más allá de los logros analíticos y explicativos del empirismo de primera y segunda generaciones, persiste en ambos una cierta debilidad conceptual para aclarar el origen de la conexión entre la polarización territorial de los actores y la centralización de los contenidos Web. Dicho de otro modo, el contexto de polarización de los actores no tiene por qué aludir de antemano a un cuadro explicativo de la centralización de contenidos. Lo anterior nos lleva quizá, una vez más, de regreso a la exploración de las estrategias de manejo de la información organizacional, la localización de las tecnoestructuras y el tipo de articulación territorial de sus poderes de decisión y acción informativa (Toudert y Buzai, 2004). Desde luego, habrá que comprobar esos supuestos, y mientras eso ocurre, el planteamiento parece reflejar un potencial explicativo para estructurar una hipótesis de trabajo a futuro.

Aclarar los motivos de la ubicación y las grandes lógicas de aglomeración de la producción Web adquiere, según nuestro punto de vista, una posición central en la discusión de la apropiación socioterritorial de las TIC en el marco de la conceptuación de la sociedad de la información. Desde esta perspectiva, resultaría quizá válido examinar la naturaleza y el significado del balance socioterritorial entre la producción y el consumo en el contexto de la articulación global-local. Dicho de otro modo, ¿tendrá sentido la sociedad de la información en un contexto dicotómico de regiones productoras y otras consumidoras de contenido?

\subsection{Dilución y pérdida del significado de los indicadores de producción de la Web}

Los indicadores de países utilizados a menudo -entre otros- por los organismos internacionales (UIT, OCDE) para analizar y explicar la apropiación de las TIC por medio de la producción Web pierden, en gran medida, sus significados una vez aplicados en el contexto de polarización que caracteriza a nuestras entidades federativas. Independientemente del tecnicismo que implica la búsqueda de una coherencia de escala en los indicadores de producción Web, no perdimos de vista que estos instrumentos de medición, lejos de reflejar números casuales y neutrales, de hecho son un sustento para la reflexión, elaboración y evaluación de políticas de desarrollo de las Tic. 
A pesar de que dos indicadores de especialización en una entidad de cien mil y otra de 10 millones de habitantes pueden arrojar un mismo valor numérico de cuatro dominios por cada mil habitantes, el poder de movilización y el impacto de 400 dominios frente 40,000 merecen considerarse como diferentes en una hipótesis de trabajo. Del mismo modo, resulta quizá necesario definir, antes que todo, con mayor objetividad el significado y el alcance de los indicadores enfocados a la elaboración del discurso de la apropiación socioterritorial de las Tic.

Como acabamos de verlo en el caso mexicano, los significados del cociente de consumo y el de especialización territorial, respectivamente, con relación a la población y a los usuarios efectivos de Internet, no son idénticos y, por lo tanto, no pueden aludir strictosensu a un mismo contenido semántico en el marco de una hipotética teoría de la apropiación socioterritorial de las TIC. Por un lado, nos enfrentamos a los antagonismos de la perspectiva socioterritorial, que reclama una integración coherente en los dispositivos analíticos y no nada más como una dimensión estigmatizante del otro; sobresale la necesidad de una adecuada transferencia semántica desde los indicadores hacia los grandes conceptos teóricos y operacionales.

Los niveles de pertinencia de los indicadores que fueron ideados en países caracterizados por una polarización extensiva son de hecho estructurados en un entorno de relativa homogeneidad de las unidades analizadas. En el caso mexicano, la polarización es intensiva y la contribución de las entidades a la producción nacional presenta fuertes desfases desde el origen mismo de los componentes intrínsecos de estos indicadores. La coherencia de los indicadores de la producción Web delimita entonces el alcance del análisis en un enfoque de escala, dejando entrever la necesidad de ajustar los instrumentos de observación a los diferentes contextos de polarización.

\section{Conclusión}

De frente al discurso emblemático de una Web que rompe con las fricciones del espacio y el tiempo camino a la consolidación de la sociedad de la información, en los hechos el desarrollo de la Web exhibe una exagerada polarización territorial. Las grandes disimetrías existentes entre los países desarrollados y los emergentes dejan apenas entrever otros padrones de polarización intranacionales que reflejan, en cierta medida, el peso de las 
jerarquías territoriales que dan sustento a las lógicas de integración (global-local).

Por su singularidad estructural y su tendencia de evolución, la polarización de la Web mexicana presenta algunas particularidades que dificultan su posicionamiento en los modelos explicativos ideados en el contexto de los países desarrollados. De antemano se presenta la necesidad de llevar a cabo estudios comparativos con el interés de sustentar o no la pertinencia de una tesis alternativa para mejorar nuestro entendimiento y acciones en el entorno de los países emergentes.

Frente a la relativa debilidad de los modelos basados en la estricta interacción de las actividades económicas para explicar la aglomeración de los actores involucrados en la producción Web, consideramos que la exploración desde el enfoque de las externalidades de tipo informativo puede ayudar a destrabar algunas incógnitas del rompecabezas locacional. Del mismo modo, tomar en cuenta los esquemas y estrategias de manejo de la información organizacional puede mejorar el acercamiento a una posible conexión entre la polarización de los actores de la Web y la centralización de los contenidos de ésta.

Todo lo anterior parece abrir un campo de acción optimista para la instrumentación de políticas de reorientación y corrección de un desarrollo que parece a todas las luces encaminarse en el sentido de agudizar la polarización existente en la "economía tradicional”.

\section{Bibliografía}

Asociación Mexicana de Internet (AMIPCI), (2000 y 2004), Hábitos del mercado en línea en México, Asociación Mexicana de Internet, www.amipci.org.mx/contenidos/estudios.html.

Bakis, Henry (1993a), "Economic and Social Geography Toward the Integration of Communications Network Studies", en Henry; Bakis, Rondal Abler y Edward Roche, (cords.), Corporate Networks, International Telecommunications and Interdependance, Belhaven Press, Londres, pp. 1-15.

Bakis, Henry (1993b), Les réseaux et leurs enjeux sociaux, col. PUF, Col. Que sais-je?, París. 
Banamex-Citigroup (2001 y 2004), México. Indicadores regionales y actividad económica, División de Estudios Económicos y Sociales, 2004, División de Estudios Económicos y Sociopolíticos, agosto 2001 y 2004, México.

Bertrand, Nathalie (2001), "Technologies d'information et de communication: quel rôle dans les dynamiques territoriales et les processus de développement, Revue d'Économie Régionale et Urbaine, 1, 135-152.

Blum, Bernardo y Avi Goldfarb (2005), Does the Internet Defy the Law of Gravity?, Working paper, www.rotman. utoronto.ca/ agoldfarb/gravity.pdf. En curso de publicación en Journal of International Economics.

Bresnahan, Timothy y Manuel Trajtenberg (1995), "General Purpose Technologies: Engines of Growth?," Journal of Econometrics, 65: 83-108.

Brunet, Roger (2001), Le déchiffrement du monde, Mappemonde, Belin, París.

Cahuc, Pierre; Hubert Kempf, y Thierry Verdier (2001), Interactions sociales et comportements économiques, Annales d'Économie et Statistiques, 63-64:1-11.

Cairncross, Frances (1997), The Death of Distance, Harvard University Press, Cambridge.

Cairncross, Frances (2001), The Death of Distance, 2.0, How the Communications Revolution will Change our Lives, Texere, Londres.

Capello, Roberta y Peter Nijkamp (1995), Le rôle des externalités de réseaux dans les performances des firmes et des régions: l'exemple des NTIC," en Alain Rallet, André y Torre,. (coords.), Economie industrielle et économie spatiale, Economica, París, pp. 272-293.

Castells, Manuel (2001), The Internet Galaxy. Reflexion on the Internet, Business, and Society, Oxford University Press, Nueva York. 
Cornu, Bernard Jean; Louis Fullsack; Yves Jeanneret; Alain Kiyindou; Sarah Labelle; Christian Le Moenne; Françoise Massit-Folléa, y M. Mathien (2005), "Vous avez dit société de l'information?", en Catherine Souyri, (coord.), La "société de l'information": glossaire critique?", La Documentation Française, París, pp. 9-35.

Damilaville, Loïc (2005), "Gouvernance de l'Internet”, en Catherine Souyri (coord.), La 'société de l'information': glossaire critique, La Documentation Française, París, pp. 80-82.

Dupuy, Gabriel (1991), "Nouvelles technologies, nouveaux territoires? pour conclure", en F. Rowe y P. Veltz, (coords.), Entreprises et territoire en réseaux, Presse de l'École National des Ponts et Chaussées, París, pp. 291-300.

Dupuy, Gabriel (1993), "Géographie et économie des réseaux”, L’espace géographique, xxII, (3):193-209.

Dodge, Martin y Narushige Shiode (2000), "Where on the Earth is the Internet? An Empirical Investigation of the Geography of the Internet”, en James Wheeler, Yuko Aoyama y Warf Barney (coords.), Cities in the Telecommunications Age: The Fracturing of Geographies, Routledge, Londres pp. 42-53.

Dodge, Martin y Rob Kitchin (2001), Mapping CyberSpace, Routledge, Londres.

Encuesta Nacional Cibermex (2000), Ciberespacio, territorio y territorialidad en México, Universidad Autónoma de Baja California, Mexicali.

Encuesta Nacional Cibermex (2004), Ciberespacio, territorio y territorialidad en México, Universidad Autónoma de Baja California, Mexicali.

Eveno, Emmanuel (1999), "Le développement local sur les TIC n'existe pas, nous l'avons rencontré”, Pouvoirs Locaux, II, (41): 58-66.

Expansión (2005), Las 500 empresas más importantes de México, Grupo Editorial Expansión, Versión digital 2005, México. 
Forman, Chris; Avi Goldfarb y Shane Greenstein (2003), "The Geographic Dispersion of Commercial Internet Use," en Steven Wildman y Lorrie Faith Cranor, Rethinking Rights and Regulations: Institutional Responses to New Communication Technologies, MIT Press, Cambridge, pp. 113-146.

Forman, Chris; Avi Goldfarb y Shane Greenstein (2005), "How did Location affect Adoption of the Commercial Internet? Global Village vs. Urban Leadership," Journal of Urban Economics, 58 (3): 389-420.

Gaspar, Jess y Edward Glaeser (1998), "Information Technology and the Future of Cities", Journal of Urban Economics, 43 (1): 136-156.

Graham, Stephen y Simon Marvin (2001), Splintering Urbanism: Networked Infraestructures Tecnological Mobilities and theUrban Conditions, Routledge, Londres.

Greenstein, Shane (2006), “The Economic Geography of Internet Infrastructure in the United States," en Martin Caves, Sumit Majumdar y Ingo Vogelsang, (coords.), The Handbook of Telecommunications Economics, vol. II, sec. II, cap. 8, Elsevier, Amsterdam.

Hillis, Ken (1999), "On the Margins: The Invisibility of Communications in Geography", Progress in Human Geography, 22 (4): 543-566.

Kolko, Jed (2000), "The Death of Cities? The Death of Distance? Evidence from the Geography of Commercial Internet Usage," en Ingo Vogelsang y Benjamin Compaine, (coords.), The Internet Upheaval, MIT Press, Cambridge, pp. 73-98.

Krymalowski, Mark David (2000), Die regionale verteilung von domainnamen in Deutschland, Tesis en Geografía Socioeconómica, Diplomado (diplomprüfung) en administración de empresas, Facultad de Estudios Socioeconómicos, Universidad de Colonia, Colonia. 
Lévy, Pierre (1997), Collective Intelligence. Mankind's Emerging World in Cyberespace, Plenum Publishing Corporation, Londres.

Lévy, Pierre (1998), Becoming Virtual. Reality in the Digital Age, Plenum Publishing Corporation, Londres.

Moss, Mitchell y Anthony Townsend (1997), "Tracking the Net: Using Domain Names to measure the Growth of the Internet in U.S. Cities", Journal of Urban Technology, 4 (3): 47-60.

Moss, Mitchell y Anthony Townsend (2000), “The Internet Backbone and the American Metropolis", Information Society, 16 (1): 35-47.

NIC-México (2002), Recopilación de estadísticas y conteos sobre nombres de dominio, hosts y servidores de Web en México y el mundo, Network Information Center México, Monterrey.

NIC-México (2005), Datos de conteo de dominios, Network Information Center México, Monterrey.

Organización para la Cooperación y el Desarrollo Económicos (OCDE) (2003), Science, technologie et industrie: Tableau de bord de l'OCDE. Versión electrónica, www.oecd.org.

Puel, Gilles y Matthieu Vidal (2003), "Le bassin de Castre-Mazamet: d'un bassin traditionnel au développement par les TIC" Revue d'Économie Régionale et Urbaine, 3: 477-498.

Saxenian, AnnaLee (1994), Regional Advantages: Cultural and Competition in Silicon Valley and Route 128, Harvard University Press, Cambridge.

Saxenian, AnnaLee (2000), "Les limites de l'autarcie: Silicon Valley et Route 128" en G. Benko y A. Lipietz, (coords.), La richesse des régions: la nouvelle géographie socio-économique, PUF, París, pp. 121-147.

Souyri, Catherine (2005), La "société de l'information": glossaire critique, La Documentation Française, París. 
Suire, Rafael y Jérôme Vicente (2002), "Géographie de la Netéconomie: qu'y a-t-il de nouveau?”, en Maurice Baslé, y Thiery Penard (coords.), Europe: la société européenne de l'information, Economica, París 87-117.

Toudert, Djamel (2000), "La www en la frontera mexicana. Hacia el descubrimiento de una dimension desconocida", Frontera Norte, 13 (24): 7-33.

Toudert, Djamel (2003), “La integración telemática en México: algunos límites y contradicciones de la planeación centralizada", Región y Sociedad, xv (28): 193-223.

Toudert, Djamel (2005), El ciberespacio, territorios y territorialidad en México, ponencia presentada en el coloquio Fronteras y Poderes en América Latina, Centro de Investigación y Documentación sobre América Latina-CREDAL-CNRS, 20-21 de junio del 2005, París.

Toudert, Djamel y Gustavo Buzai (2004), Cibergeografía. Tecnologías de la información y las comunicaciones (TIC) en las nuevas visiones espaciales, Universidad Autónoma de Baja California, Baja California.

Veltz, Pierre (2002), Des lieux et des liens, politique du territoire à l'heure de la mondialisation, Édition de l'Aube, París.

Vicente, Jérôme (2002), "Externalités de réseaux vs. externalités informationnelles dans les dynamiques de localisation", Revue d'Economie Regionale et Urbaine, 4, 535-552.

Wolton, Dominique (2000), Internet et aprés? Une théorie critique des nouveaux médias, Flamarion, París.

Zimmermann, Jean-Benoit (2001), “The Firm-Territory Relationships in the Globalization: Towards a New Rational", European Journal of Economic and Social Systems, 15: 57-75.

Zook, Matthew (2000), "Internet Metrics: Using Hosts and Domain Counts to Map the Internet Globally", Telecommunications Policy, 24 (6-7): 613-620. 
Zook, Matthew (2001), “Old Hierarchies or New Networks of Centrality? The Global Geography of the Internet Content Market", American Behavioral Scientist, 44 (10): 16791696.

Zook, Matthew (2005), The Geography of the Internet Industry: Venture Capital, Dot-coms, and Local Knowledge, Blackwell Publishers, Massachusetts.

Recibido: 9 de enero de 2006. Aceptado: 29 de mayo de 2006.

Djamel Toudert es doctor en Geografía, Urbanismo y Ordenamiento Territorial por el Instituto de Altos Estudios de América Latina, Universidad de París III. Actualmente es investigador del Instituto de Investigaciones Sociales de la UABC; coordinador general de la Cooperativa de Enseñanza y Aprendizaje Geográfico Integral (http://www.ceagi.org) y consultor en tecnologías de la información geográfica. Su línea de investigación actual es la apropiación socioterritorial de las Tecnologías de la Información y las Comunicaciones (TIC). Sus publicaciones más recientes son Cibergeografía. Tecnologías de la información y las comunicaciones (TIC) en las nuevas visiones espaciales, Edición de la Universidad Autónoma de Baja California, Baja California; "Contribución al estudio de la articulación entre telefonía fija doméstica y marginación socioterritorial en las localidades de los estados de la Frontera Norte Mexicana", Frontera Norte, Revista del Colegio de la Frontera Norte, vol. 15, núm. 30, pp. 7-32; "La integración telemática en México: algunos límites y contradicciones de la planeación centralizada, Región y Sociedad", Revista del Colegio de Sonora, vol. xv, núm. 28, pp. 193-223; "La www en la frontera mexicana. Hacia el descubrimiento de una dimension desconocida", Frontera Norte, Revista del Colegio de la Frontera Norte, vol. 13, núm. 24, pp. 7-33. 